\title{
The Faculty of Engineering AtTribute Assessment Process at The University OF MANitoba: SUGGESTIONS FOR CLOSING THE LOOP
}

\author{
Jillian Seniuk Cicek, Sandra Ingram and Nariman Sepehri \\ Faculty of Engineering, University of Manitoba, Winnipeg, MB, Canada R3T 5V6 \\ umseniuk@myumanitoba.ca
}

\begin{abstract}
This paper describes the findings from a three-year longitudinal study at the University of Manitoba designed to explore how the Canadian Engineering Accreditation Board (CEAB) graduate attributes are manifested and measured in the Faculty of Engineering's curriculum. Instructors from the Departments of Biosystems, Civil, Mechanical, and Electrical and Computer Engineering were asked to consider the presence of four of the 12 CEAB attributes and their subsequent indicators in one engineering course taught in one academic year. Each year, four different attributes were targeted, chosen to reflect both the traditional/technical and the professional/workplace competencies. Data were collected using a selfadministered checklist, which evolved over the three years of the study in an effort to more clearly define student attribute competency levels, and to develop a common language and understanding in regards to the graduate attributes and the process of outcomes-based assessment. This final phase of the study enables us to understand how all 12 of the CEAB graduate attributes are manifest and measured across our engineering curricula, to discuss our findings within the context of outcomes-based assessment and accreditation protocols, and to strategize ways to close the loop.
\end{abstract}

Keywords: $\quad$ CEAB graduate attribute assessment; accreditation; instructor checklist

\section{INTRODUCTION}

According to Suskie (2008), "Assessments should yield value that justifies the time and expense we put into them... They should not take so much time that they detract from other essential activities such as teaching" [1]. Understanding what comprises best practices for assessment enables faculty to optimize its value. Poignantly, the overriding best practice for assessment is simple: It should be good. There are five characteristics of 'good' assessment that have emerged from the literature in recent years: (1) good assessments are used; (2) good assessments are economical, particularly in terms of time; (3) good assessments provide reasonably accurate and truthful results (reliable and valid); (4) good assessments are valued; and (5) good assessments result from, and target, specific and important goals [1].

Increasing the value of assessments is especially important in engineering education, as assessment is linked to accountability and accreditation. It is also essential because in countless ways, assessment is distasteful to many educators. In fact, for some, assessment may be considered the dark horse of education. Testimonial to this is the fact that the term was avoided for a number of years:

Assessment permeates every aspect of our lives, and is a natural and automatic activity (Rowntree, 1987, p. 4). In the educational context, the terrors evoked by the term 'assessment' have distorted its necessity, centrality and its potentially neutral position. Indeed 'assessment' is considered so negative that the term 'evaluation' was preferred for many years. [2]

The idea of asking faculty to perform additional assessments, on top of their own individual course assessments, as part of the process for program improvement and accreditation, increases assessment distaste. However, program assessment is required and essential for improving program quality and thereby, student learning. Consequently, it is most beneficial to make assessment as painless as possible: it must be clearly understood, purposed, transparent, efficient and effective, which will help develop trust and create faculty buy-in [3][4][5]. It is through the efficiency and effectiveness of the assessment process that an appropriate middle ground can be found to generate the desired outcome, i.e., improvement of teaching and learning for a reasonable and sustainable degree of effort. When this happens, stakeholders will be satisfied, and a culture of assessment will be embedded in the program [1]. 


\section{BACKGROUND}

In Fall 2011, inspired by the new accreditation requirements for $\mathrm{CEAB}$, which mandated a continuous curriculum improvement cycle informed by the outcomesbased assessment data of the graduate attributes [5], the Faculty of Engineering at the University of Manitoba began a three-year longitudinal faculty attribute assessment study. The objectives of the study were to determine how the $12 \mathrm{CEAB}$ graduate attributes were manifest and measured in the four engineering departments within the faculty: Biosystems, Civil, Electrical and Computer (ECE), and Mechanical, and to explore the extent to which the assessed attributes resulted in course content proficiency [6]. Additionally, informed by the analysis of the data after the first year of the study, we began to investigate what instructors determine as the level that represents student competency for each attribute/indicator [7].

Each year, a different group of instructors were asked to complete a self-administered checklist that was designed to determine which attribute indicators instructors assessed or demonstrated in their courses, and which indicators they did not. Assessed indicators were defined as linked to instructor's assessment tools, such as quizzes, assignments, projects, labs or exams. In the second part of the checklist, instructors were asked to map each assessed indicator to one of their assessment tools, and specify how they communicated the assessment results to students, i.e., numerical marks, letter grades, rubrics or comments. Demonstrated indicators were ones that instructors felt were covered within the course content, but that were not formally assessed. Indicators that instructors neither assessed nor demonstrated were also categorized.

For every year of the study, the Heads for each department gave a list of potential instructor participants to the researchers. Generally, each instructor was only asked to participate in the study once (although there were exceptions where a few instructors were unintentionally asked to participate twice). Participating instructors completed the checklist for one course during either the Fall or Winter semester. Thereby, data were collected twice each academic year. For each year of the study, four different attributes were targeted, so that by the end of the three years, all 12 attributes were investigated within the faculty. The initial division of the attributes was determined by choosing two technical skills and two professional skills [6][7]. Tables 1 and 2 show how the attributes were divided, and which courses were investigated.

At the end of each year, we analyzed the data and disseminated our findings to the participating instructors, Department Heads, and the Faculty's curriculum management committee (CMC). We have also presented our findings at three annual CEEA conferences [6][7][8], and published the findings from the second year of the study in the International Journal of Engineering Education [9]. The intent has been to give each department data that can be used in their cycle of continual program improvement.

This paper is the last installment of this study. All three years of the research data have been collected and analyzed. As there is a large amount of data, only a portion can be reported in this forum. Therefore, for this paper, we will present the data from Part A of the checklist, specifically the results for which indicators are assessed, demonstrated or not demonstrated. The findings will be contextualized for each engineering department individually, so that they may be used to inform the departments' individual assessment processes.

\section{METHODS}

For each year of the faculty attribute assessment study, four of the $12 \mathrm{CEAB}$ graduate attributes and their associated foci and indicators as conceived by the CMC were built into the checklist administered during the 2011-12, 2012-13 and 2013-14 academic years. A selection of instructors who had ideally not participated in the study before and who were chosen by their program department heads were asked to report the extent to which the indicators for each attribute were built into their course and its associated mark distribution in Part A of the checklist (Full, Part, None). If the indicator was marked as Full, then instructors were asked to record the assessment tools, assessment communication and the expected competency level and target percentage for the indicator [8]. In Part B of the checklist, instructors were asked to record course assessment results.

Over the progression of the study, there were changes made to the checklist, including amendments to (i) the language, to more accurately reflect the language of outcomes-based assessment; (ii) the reporting categories, where a Target Percentage category was added to encourage instructors to set a goal for the minimum number of students in class whom they felt should perform at the level of expected competency, which was proposed to add another layer to the process of outcomesbased assessment; (iii) the structure and content, to make the checklist more user-friendly to support instructor participation; and (iv) the process by which the checklist was introduced to instructors. As confusion and the rate of data return were found to be issues in the previous iterations of the study, a workshop was instituted in an attempt to circumvent these [8].

The checklist was offered to instructors via email, and in the last year of the study, via a workshop in October of each year. Part A of the checklist was self-administered by instructors at the beginning and through the middle of the semester, and Part B was completed once course assessments were finalized (December to mid-January). 
Table 1: Faculty attribute assessment study: Biosystems (BIOE) and Civil (CIVL) engineering courses assessed over 3 years.

\begin{tabular}{|c|c|c|c|}
\hline YEAR & ATTRIBUTES & BIOE (11) & CIVL(10) \\
\hline $\begin{array}{l}2011- \\
2012\end{array}$ & $\begin{array}{l}\text { 3. Investigation } \\
\text { 4. Design } \\
\text { 8. Professionalism } \\
\text { 12. Lifelong } \\
\quad \text { Learning }\end{array}$ & $\begin{array}{l}\text { BIOE } 3580 \text { - Design } \\
\text { Trilogy II } \\
\text { BIOE } 4580 \text { - Design } \\
\text { Trilogy III }\end{array}$ & $\begin{array}{l}\text { CIVL } 3760 \text {-Structural } \\
\text { Analysis } \\
\text { CIVL } 4030 \text {-Structural } \\
\text { Design } 3\end{array}$ \\
\hline $\begin{array}{l}2012- \\
2013\end{array}$ & $\begin{array}{l}\text { 2. Problem } \\
\text { Analysis } \\
\text { 5. Use of Eng. } \\
\text { Tools } \\
\text { 7. Communication } \\
\text { Skills } \\
\text { 10. Ethics and } \\
\text { Equity }\end{array}$ & $\begin{array}{l}\text { BIOE } 2580 \text { - Design } \\
\text { Trilogy I } \\
\text { BIOE } 3320 \text { - Eng. } \\
\text { Properties of } \\
\text { Biological Material } \\
\text { BIOE } 3590 \text { - } \\
\text { Mechanics of } \\
\text { Materials in } \\
\text { Biosystems } \\
\text { BIOE } 4520 \text { - } \\
\text { Crop Preservation }\end{array}$ & $\begin{array}{l}\text { CIVL } 3730 \text { - } \\
\text { Geotechnical } \\
\text { Materials \& Analysis } \\
\text { CIVL } 3760 \text {-Structural } \\
\text { Analysis } \\
\text { CIVL } 1440 \text { - Intro to } \\
\text { Statics }\end{array}$ \\
\hline $\begin{array}{l}2013- \\
2014\end{array}$ & $\begin{array}{l}\text { 1. A Knowledge } \\
\text { Base for } \\
\text { Engineering } \\
\text { 6. Individual \& } \\
\text { Team Work } \\
\text { 9. Impact of } \\
\text { Engineering } \\
\text { on Society \& } \\
\text { the Environment } \\
\text { 11. Economics \& } \\
\text { Project } \\
\text { Management }\end{array}$ & $\begin{array}{l}\text { BIOE } 2590 \text { - Biology } \\
\text { for Engineers } \\
\text { BIOE } 4240- \\
\text { Graduation Project } \\
\text { BIOE } 3320- \\
\text { Eng. Properties of } \\
\text { Biological Materials } \\
\text { BIOE } 4240 \text { - } \\
\text { Graduation Project } \\
\text { AGRI } 2200 \text { - } \\
\text { Principles of Plant \& } \\
\text { Animal Physiology }\end{array}$ & $\begin{array}{l}\text { CIVL 2840 - } \\
\text { Geomatics } \\
\text { CIVL } 3750- \\
\text { Hydrology } \\
\text { CIVL 4380 - } \\
\text { Elements of Law for } \\
\text { Civil Engineers } \\
\text { CIVL 4050 - } \\
\text { Engineering } \\
\text { Economics } \\
\text { CIVL 4470 - } \\
\text { Watershed } \\
\text { Processes }\end{array}$ \\
\hline
\end{tabular}

Table 2: Faculty attribute assessment study: Electrical \& Computer (ECE) and Mechanical (MECH) engineering courses assessed over 3 years.

\begin{tabular}{|c|c|c|c|}
\hline YEAR & ATTRIBUTES & ECE (9) & MECH (11) \\
\hline $\begin{array}{l}2011- \\
2012\end{array}$ & $\begin{array}{l}\text { 3. Investigation } \\
\text { 4. Design } \\
\text { 8. Professionalism } \\
\text { 12. Lifelong } \\
\quad \text { Learning }\end{array}$ & $\begin{array}{l}\text { ECE } 3720 \text { - } \\
\text { Introductory } \\
\text { Power \& Machines } \\
\text { ECE } 4310 \text { - Electrical } \\
\text { Energy Systems } 2\end{array}$ & $\begin{array}{l}\text { MECH 3980 - } \\
\text { Mech Eng'g Lab } \\
\text { MECH 4182-- } \\
\text { Aerospace Structures: } \\
\text { Analysis \& Design }\end{array}$ \\
\hline $\begin{array}{l}2012- \\
2013\end{array}$ & $\begin{array}{l}\text { 2. Problem } \\
\text { Analysis } \\
\text { 5. Use of Eng. } \\
\text { Tools } \\
\text { 7. Communication } \\
\text { Skills } \\
\text { 10. Ethics and } \\
\text { Equity }\end{array}$ & $\begin{array}{l}\text { ECE } 3740 \text { - Systems } \\
\text { Engineering Principles } \\
\text { ECE } 3600 \text { - Physical } \\
\text { Electronics } \\
\text { ECE } 4260 \text { - } \\
\text { Communication } \\
\text { Systems }\end{array}$ & $\begin{array}{l}\text { ENG } 1460 \text { - Intro. to } \\
\text { Thermal Sciences } \\
\text { MECH } 4510- \\
\text { Fundamentals of } \\
\text { Finite Element } \\
\text { Analysis } \\
\text { MECH } 3550 \text { - } \\
\text { Robotics \& Computer } \\
\text { Numerical Control } \\
\text { MECH } 2272- \\
\text { Engineering Materials } \\
\text { MECH } 4412- \\
\text { Heating, Ventilation } \\
\text { \& Air Conditioning }\end{array}$ \\
\hline $\begin{array}{l}\text { 2013- } \\
2014\end{array}$ & $\begin{array}{l}\text { 1. A Knowledge } \\
\text { Base for } \\
\text { Engineering } \\
\text { 6. Individual \& } \\
\text { Team Work } \\
\text { 9. Impact of } \\
\text { Engineering } \\
\text { on Society \& } \\
\text { the Environment } \\
\text { 11. Economics \& } \\
\text { Project } \\
\text { Management }\end{array}$ & $\begin{array}{l}\text { ECE } 3670 \text { - } \\
\text { Electronics 3E } \\
\text { ECE } 2240 \text {-Numerical } \\
\text { Methods for } \\
\text { Engineers } \\
{ }^{*} \text { ENG } 1450 \text { - Intro. to } \\
\text { Electrical \& Computer } \\
\text { Engineering } \\
\text { **ECE } 4600 \text { - Group } \\
\text { Design Project }\end{array}$ & $\begin{array}{l}\text { MECH } 2202- \\
\text { Thermodynamics } \\
\text { MECH } 4452- \\
\text { Aircraft Performance, } \\
\text { Dynamics \& Design } \\
\text { MECH } 2272- \\
\text { Engineering Materials } \\
\text { MECH } 3170- \\
\text { Project Management }\end{array}$ \\
\hline
\end{tabular}

${ }^{*}$ Completed checklist for Individual \& Teamwork. ${ }^{* *}$ Completed checklist for Impact of Eng. and Eco. \& Project Management.

\section{FINDINGS}

There were 41 courses for which instructors completed or partially completed a faculty attribute assessment checklist: 11, 10, 9 and 11 courses each from the departments of Biosystems, Civil, ECE and Mechanical engineering (Tables 1 and 2). Partial completions of the checklist were found in two usages. In one department, two instructors focused on realizing the checklist for one and two of the four attributes respectively, a decision that supported their department's assessment protocol. Data from these two checklists are still used in the study, for although they may not give a complete picture of all four targeted attributes in those particular courses, the data that are reported are still valuable. Those courses can be explored further by the department, dependent on their assessment goals. The second type of incomplete checklist was found when the assessment results portion of the checklist (Part B) was left unfinished. Those missing data do not affect the findings for this paper, as only data from Part A of the checklist will be presented.

For Part A of the checklist, instructors were asked to consider the four targeted attributes and their associated indicators, and report on whether those indicators were built into the associated mark distribution in the course (Full), if the indicator was demonstrated but there was no formal process built into the mark distribution (Part), or if the content of the course did not demonstrate the indicator (None). The following four sections report the results by individual engineering departments.

\subsection{Attribute/Indicator Checklist Results: Biosystems}

Table 3 and Figure 1 show the assessment results for the attributes/indicators in 11 courses in the Biosystems Engineering program, as collected over the three years that the study was implemented.

Table 3: Number and percentage of attribute indicators assessed for 11 courses in Biosystems Eng., 2011-2014.

\begin{tabular}{|c|c|c|c|}
\hline \multicolumn{4}{|c|}{ BIOSYSTEMS (11) } \\
\hline & Full & Part & None \\
\hline $\begin{array}{l}\text { 1. Knowledge Base for } \\
\text { Engineering - } 7 \text { indicators }\end{array}$ & $\begin{array}{c}6 \\
85.7 \%\end{array}$ & $\begin{array}{c}1 \\
14.3 \%\end{array}$ & $\begin{array}{c}0 \\
0 \%\end{array}$ \\
\hline $\begin{array}{l}\text { 2. Problem Analysis - } 13 \\
\text { indicators }\end{array}$ & $\begin{array}{c}10 \\
76.9 \%\end{array}$ & $\begin{array}{c}3 \\
23.1 \%\end{array}$ & $\begin{array}{c}0 \\
0 \%\end{array}$ \\
\hline $\begin{array}{l}\text { 3. Investigation - } 28 \\
\text { indicators }\end{array}$ & $\begin{array}{c}8 \\
28.6 \%\end{array}$ & $\begin{array}{c}13 \\
46.4 \%\end{array}$ & $\begin{array}{c}7 \\
25 \%\end{array}$ \\
\hline 4. Design - 31 indicators & $\begin{array}{c}22 \\
71 \%\end{array}$ & $\begin{array}{c}8 \\
25.8 \%\end{array}$ & $\begin{array}{c}1 \\
3.2 \% \\
\end{array}$ \\
\hline $\begin{array}{l}\text { 5. Use of Engineering } \\
\text { Tools - } 10 \text { indicators }\end{array}$ & $\begin{array}{c}4 \\
40 \%\end{array}$ & $\begin{array}{c}2 \\
20 \%\end{array}$ & $\begin{array}{c}4 \\
40 \%\end{array}$ \\
\hline $\begin{array}{l}\text { 6. Individual \& Teamwork - } \\
16 \text { indicators }\end{array}$ & $\begin{array}{c}1 \\
6.3 \%\end{array}$ & $\begin{array}{c}5 \\
31.3 \%\end{array}$ & $\begin{array}{c}10 \\
62.5 \%\end{array}$ \\
\hline $\begin{array}{l}\text { 7. Communication Skills - } \\
18 \text { indicators }\end{array}$ & $\begin{array}{c}8 \\
44.4 \%\end{array}$ & $\begin{array}{c}6 \\
33.3 \%\end{array}$ & $\begin{array}{c}4 \\
22.2 \%\end{array}$ \\
\hline $\begin{array}{l}\text { 8. Professionalism - } 13 \\
\text { indicators }\end{array}$ & $\begin{array}{c}4 \\
30.8 \% \\
\end{array}$ & $\begin{array}{c}9 \\
69.2 \%\end{array}$ & $\begin{array}{c}0 \\
0 \%\end{array}$ \\
\hline $\begin{array}{l}\text { 9. Impact of Engineering } \\
\text { on Society } \& \text { the } \\
\text { Environment - } 8 \text { indicators }\end{array}$ & $\begin{array}{c}0 \\
0 \%\end{array}$ & $\begin{array}{c}3 \\
37.5 \%\end{array}$ & $\begin{array}{c}5 \\
62.5 \%\end{array}$ \\
\hline $\begin{array}{l}\text { 10. Ethics \& Equity } \\
9 \text { indicators }\end{array}$ & $\begin{array}{c}3 \\
33.3 \%\end{array}$ & $\begin{array}{c}1 \\
11.1 \%\end{array}$ & $\begin{array}{c}5 \\
55.5 \%\end{array}$ \\
\hline $\begin{array}{l}\text { 11. Economics \& Project } \\
\text { Management - } 18 \\
\text { indicators }\end{array}$ & $\begin{array}{c}6 \\
33.3 \%\end{array}$ & $\begin{array}{c}8 \\
44.4 \%\end{array}$ & $\begin{array}{c}4 \\
22.2 \%\end{array}$ \\
\hline $\begin{array}{l}\text { 12. Lifelong Learning - } 27 \\
\text { indicators }\end{array}$ & $\begin{array}{c}7 \\
25.9 \%\end{array}$ & $\begin{array}{c}10 \\
37 \%\end{array}$ & $\begin{array}{c}10 \\
37 \%\end{array}$ \\
\hline
\end{tabular}




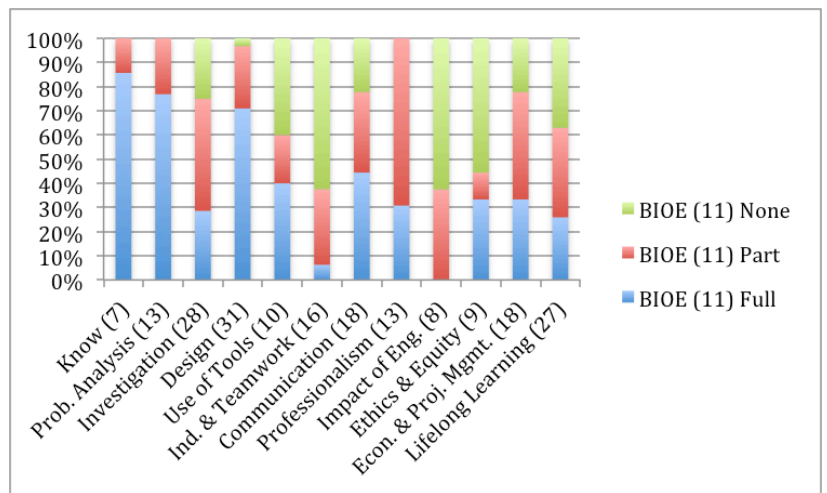

Fig. 1. Percentage of CEAB Graduate Attribute indicators assessed (Full), demonstrated (Part) and undemonstrated

(None) in 11 courses in the Biosystems Engineering program, 2011-14.

\subsection{Attribute/Indicator Checklist Results: Civil}

Table 4 and Figure 2 show the assessment results for the attributes/indicators in 10 courses in the Civil Engineering program, as collected over the three years that the study was implemented.

Table 4: Number and percentage of attribute indicators assessed for 10 courses in Civil Eng., 2011-2014.

\begin{tabular}{|c|c|c|c|}
\hline \multicolumn{4}{|c|}{ CIVIL (10) } \\
\hline & Full & Part & None \\
\hline $\begin{array}{l}\text { 1. Knowledge Base for } \\
\text { Engineering - } 7 \text { indicators }\end{array}$ & $\begin{array}{c}7 \\
100 \% \\
\end{array}$ & $\begin{array}{c}0 \\
0 \% \\
0\end{array}$ & $\begin{array}{c}0 \\
0 \% \\
0\end{array}$ \\
\hline $\begin{array}{l}\text { 2. Problem Analysis - } 13 \\
\text { indicators }\end{array}$ & $\begin{array}{c}11 \\
84.6 \%\end{array}$ & $\begin{array}{c}1 \\
7.7 \% \\
\end{array}$ & $\begin{array}{c}1 \\
7.7 \% \\
\end{array}$ \\
\hline $\begin{array}{l}\text { 3. Investigation - } 28 \\
\text { indicators }\end{array}$ & $\begin{array}{c}16 \\
57.1 \%\end{array}$ & $\begin{array}{c}5 \\
17.9 \%\end{array}$ & $\begin{array}{c}7 \\
25 \% \\
\end{array}$ \\
\hline 4. Design - 31 indicators & $\begin{array}{c}16 \\
51.6 \%\end{array}$ & $\begin{array}{c}4 \\
12.9 \%\end{array}$ & $\begin{array}{c}11 \\
35.5 \%\end{array}$ \\
\hline $\begin{array}{l}\text { 5. Use of Engineering } \\
\text { Tools - } 10 \text { indicators }\end{array}$ & $\begin{array}{c}6 \\
60 \% \\
\end{array}$ & $\begin{array}{c}1 \\
10 \% \\
\end{array}$ & $\begin{array}{c}3 \\
30 \% \\
\end{array}$ \\
\hline $\begin{array}{l}\text { 6. Individual \& Teamwork - } \\
16 \text { indicators }\end{array}$ & $\begin{array}{c}7 \\
43.8 \% \\
\end{array}$ & $\begin{array}{c}7 \\
43.8 \% \\
\end{array}$ & $\begin{array}{c}2 \\
12.5 \% \\
\end{array}$ \\
\hline $\begin{array}{c}\text { 7. Communication Skills - } \\
18 \text { indicators }\end{array}$ & $\begin{array}{c}10 \\
55.6 \%\end{array}$ & $\begin{array}{c}1 \\
5.6 \% \\
\end{array}$ & $\begin{array}{c}7 \\
38.9 \%\end{array}$ \\
\hline $\begin{array}{l}\text { 8. Professionalism - } 13 \\
\text { indicators }\end{array}$ & $\begin{array}{c}3 \\
23.1 \% \\
\end{array}$ & $\begin{array}{c}3 \\
23.1 \% \\
\end{array}$ & $\begin{array}{c}7 \\
53.8 \% \\
\end{array}$ \\
\hline $\begin{array}{l}\text { 9. Impact of Engineering } \\
\text { on Society \& the } \\
\text { Environment - } 8 \text { indicators }\end{array}$ & $\begin{array}{c}5 \\
62.5 \%\end{array}$ & $\begin{array}{c}3 \\
37.5 \%\end{array}$ & $\begin{array}{c}0 \\
0 \%\end{array}$ \\
\hline $\begin{array}{r}\text { 10. Ethics \& Equity } \\
9 \text { indicators }\end{array}$ & $\begin{array}{c}1 \\
1.1 \% \\
\end{array}$ & $\begin{array}{c}0 \\
0 \% \\
\end{array}$ & $\begin{array}{c}8 \\
88.9 \% \\
\end{array}$ \\
\hline $\begin{array}{l}\text { 11. Economics \& Project } \\
\text { Management - } 18 \\
\text { indicators }\end{array}$ & $\begin{array}{c}13 \\
72.2 \%\end{array}$ & $\begin{array}{c}4 \\
22.2 \%\end{array}$ & $\begin{array}{c}1 \\
5.6 \%\end{array}$ \\
\hline $\begin{array}{l}\text { 12. Lifelong Learning - } 27 \\
\text { indicators }\end{array}$ & $\begin{array}{c}0 \\
0 \% \\
\end{array}$ & $\begin{array}{c}12 \\
44.4 \% \\
\end{array}$ & $\begin{array}{c}15 \\
55.6 \%\end{array}$ \\
\hline
\end{tabular}

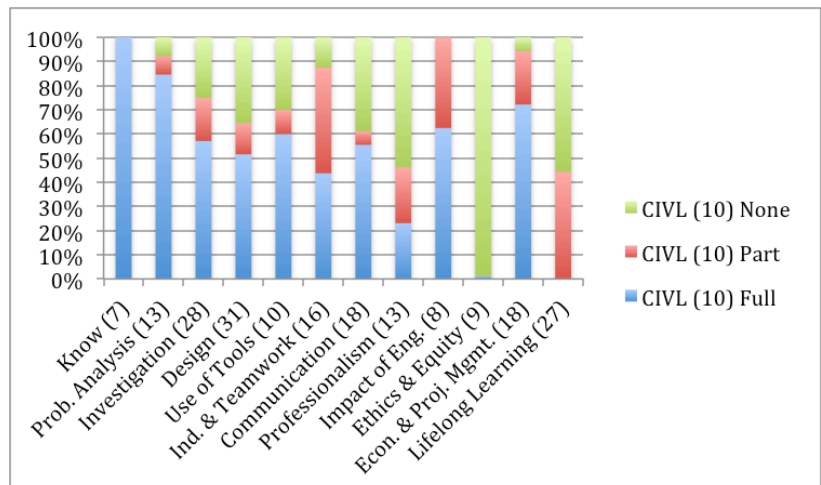

Fig. 2. Percentage of CEAB Graduate Attribute indicators assessed (Full), demonstrated (Part) and undemonstrated

(None) in 10 courses in the Civil Engineering program, 2011-14.

\subsection{Attribute/Indicator Checklist Results: ECE}

Table 5 and Figure 3 show the assessment results for the attributes/indicators in 9 courses in the Electrical and Computer Engineering program, as collected over the three years that the study was implemented.

Table 5: Number and percentage of attribute indicators assessed for 9 courses in Electrical \& Computer Eng., 2011-2014.

\begin{tabular}{|c|c|c|c|}
\hline \multicolumn{4}{|c|}{ ELECTRICAL \& COMPUTER (9) } \\
\hline & Full & Part & None \\
\hline $\begin{array}{l}\text { 1. Knowledge Base for } \\
\text { Engineering }-7 \text { indicators }\end{array}$ & $\begin{array}{c}4 \\
57.1 \%\end{array}$ & $\begin{array}{c}3 \\
42.9 \%\end{array}$ & $\begin{array}{c}0 \\
0 \%\end{array}$ \\
\hline $\begin{array}{l}\text { 2. Problem Analysis - } 13 \\
\text { indicators }\end{array}$ & $\begin{array}{c}12 \\
92.3 \%\end{array}$ & $\begin{array}{c}1 \\
7.7 \%\end{array}$ & $\begin{array}{c}0 \\
0 \%\end{array}$ \\
\hline $\begin{array}{c}\text { 3. Investigation - } 28 \\
\text { indicators }\end{array}$ & $\begin{array}{c}17 \\
60.7 \% \\
\end{array}$ & $\begin{array}{c}10 \\
35.7 \% \\
\end{array}$ & $\begin{array}{c}1 \\
3.6 \% \\
\end{array}$ \\
\hline 4. Design - 31 indicators & $\begin{array}{c}15 \\
48.4 \%\end{array}$ & $\begin{array}{c}9 \\
29 \%\end{array}$ & $\begin{array}{c}7 \\
22.6 \%\end{array}$ \\
\hline $\begin{array}{l}\text { 5. Use of Engineering } \\
\text { Tools - } 10 \text { indicators }\end{array}$ & $\begin{array}{c}6 \\
60 \% \\
\end{array}$ & $\begin{array}{c}1 \\
10 \%\end{array}$ & $\begin{array}{c}3 \\
30 \% \\
\end{array}$ \\
\hline $\begin{array}{l}\text { 6. Individual \& Teamwork - } \\
16 \text { indicators }\end{array}$ & $\begin{array}{c}6 \\
37.5 \% \\
\end{array}$ & $\begin{array}{c}6 \\
37.5 \%\end{array}$ & $\begin{array}{c}4 \\
25 \%\end{array}$ \\
\hline $\begin{array}{c}\text { 7. Communication Skills - } \\
18 \text { indicators }\end{array}$ & $\begin{array}{c}0 \\
0 \%\end{array}$ & $\begin{array}{c}6 \\
33.3 \%\end{array}$ & $\begin{array}{c}12 \\
66.7 \%\end{array}$ \\
\hline $\begin{array}{l}\text { 8. Professionalism - } 13 \\
\text { indicators }\end{array}$ & $\begin{array}{c}1 \\
7.7 \% \\
\end{array}$ & $\begin{array}{c}10 \\
77 \% \\
\end{array}$ & $\begin{array}{c}2 \\
15.4 \% \\
\end{array}$ \\
\hline $\begin{array}{l}\text { 9. Impact of Engineering } \\
\text { on Society } \& \text { the } \\
\text { Environment - } 8 \text { indicators }\end{array}$ & $\begin{array}{c}2 \\
25 \%\end{array}$ & $\begin{array}{c}3 \\
37.5 \%\end{array}$ & $\begin{array}{c}3 \\
37.5 \%\end{array}$ \\
\hline $\begin{array}{r}\text { 10. Ethics \& Equity } \\
9 \text { indicators }\end{array}$ & $\begin{array}{c}0 \\
0 \%\end{array}$ & $\begin{array}{c}2 \\
22.2 \%\end{array}$ & $\begin{array}{c}7 \\
77.8 \%\end{array}$ \\
\hline $\begin{array}{l}\text { 11. Economics \& Project } \\
\text { Management - } 18 \\
\text { indicators }\end{array}$ & $\begin{array}{c}12 \\
66.7 \%\end{array}$ & $\begin{array}{c}5 \\
27.8 \%\end{array}$ & $\begin{array}{c}1 \\
5.6 \%\end{array}$ \\
\hline $\begin{array}{l}\text { 12. Lifelong Learning - } 27 \\
\text { indicators }\end{array}$ & $\begin{array}{c}1 \\
3.7 \%\end{array}$ & $\begin{array}{c}14 \\
51.9 \%\end{array}$ & $\begin{array}{c}12 \\
44.4 \%\end{array}$ \\
\hline
\end{tabular}




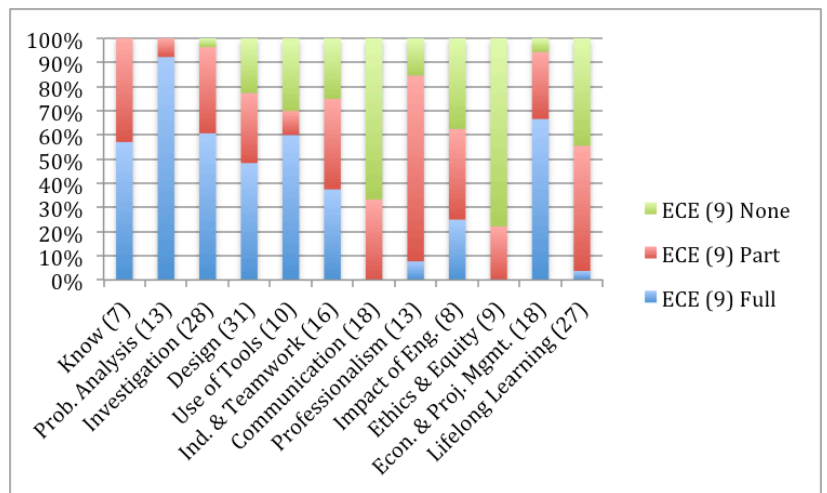

Fig. 3. Percentage of CEAB Graduate Attribute indicators assessed (Full), demonstrated (Part) and undemonstrated

(None) in 9 courses in the Electrical \& Computer Engineering program, 2011-14.

\subsection{Attribute/Indicator Checklist Results: Mechanical}

Table 6 and Figure 4 show the assessment results for the attributes/indicators in 11 courses in the Mechanical Engineering program, as collected over the three years that the study was implemented.

Table 6: Number and percentage of attribute indicators assessed for 11 courses in Mechanical Eng., 2011-2014.

\begin{tabular}{|c|c|c|c|}
\hline \multicolumn{4}{|c|}{ MECHANICAL (11) } \\
\hline & Full & Part & None \\
\hline $\begin{array}{l}\text { 1. Knowledge Base for } \\
\text { Engineering - } 7 \text { indicators }\end{array}$ & $\begin{array}{c}7 \\
100 \% \\
\end{array}$ & $\begin{array}{c}0 \\
0 \% \\
0 \%\end{array}$ & $\begin{array}{c}0 \\
0 \% \\
0 \%\end{array}$ \\
\hline $\begin{array}{l}\text { 2. Problem Analysis - } 13 \\
\text { indicators }\end{array}$ & $\begin{array}{c}13 \\
100 \%\end{array}$ & $\begin{array}{c}0 \\
0 \%\end{array}$ & $\begin{array}{c}0 \\
0 \%\end{array}$ \\
\hline $\begin{array}{l}\text { 3. Investigation - } 28 \\
\text { indicators }\end{array}$ & $\begin{array}{c}20 \\
71.4 \%\end{array}$ & $\begin{array}{c}7 \\
25.0 \%\end{array}$ & $\begin{array}{c}1 \\
3.6 \%\end{array}$ \\
\hline 4. Design - 31 indicators & $\begin{array}{c}14 \\
45.2 \% \\
\end{array}$ & $\begin{array}{c}14 \\
45.2 \% \\
\end{array}$ & $\begin{array}{c}3 \\
9.7 \%\end{array}$ \\
\hline $\begin{array}{l}\text { 5. Use of Engineering } \\
\text { Tools - } 10 \text { indicators }\end{array}$ & $\begin{array}{c}6 \\
60 \%\end{array}$ & $\begin{array}{c}3 \\
30 \%\end{array}$ & $\begin{array}{c}1 \\
10 \%\end{array}$ \\
\hline $\begin{array}{l}\text { 6. Individual \& Teamwork - } \\
16 \text { indicators }\end{array}$ & $\begin{array}{c}8 \\
50 \%\end{array}$ & $\begin{array}{c}7 \\
43.8 \%\end{array}$ & $\begin{array}{c}1 \\
6.3 \%\end{array}$ \\
\hline $\begin{array}{c}\text { 7. Communication Skills - } \\
18 \text { indicators }\end{array}$ & $\begin{array}{c}4 \\
22.2 \% \\
\end{array}$ & $\begin{array}{c}7 \\
38.9 \% \\
\end{array}$ & $\begin{array}{c}7 \\
38.9 \% \\
\end{array}$ \\
\hline $\begin{array}{l}\text { 8. Professionalism - } 13 \\
\text { indicators }\end{array}$ & $\begin{array}{c}4 \\
30.8 \% \\
\end{array}$ & $\begin{array}{c}7 \\
53.8 \% \\
\end{array}$ & $\begin{array}{c}2 \\
15.4 \% \\
\end{array}$ \\
\hline $\begin{array}{l}\text { 9. Impact of Engineering } \\
\text { on Society \& the } \\
\text { Environment - } 8 \text { indicators }\end{array}$ & $\begin{array}{c}3 \\
37.5 \%\end{array}$ & $\begin{array}{c}4 \\
50 \%\end{array}$ & $\begin{array}{c}1 \\
12.5 \%\end{array}$ \\
\hline $\begin{array}{r}10 . \text { Ethics \& Equity } \\
9 \text { indicators }\end{array}$ & $\begin{array}{c}0 \\
0 \% \\
0 \%\end{array}$ & $\begin{array}{c}3 \\
33.3 \% \\
\end{array}$ & $\begin{array}{c}6 \\
66.7 \% \\
\end{array}$ \\
\hline $\begin{array}{l}\text { 11. Economics \& Project } \\
\text { Management - } 18 \\
\text { indicators }\end{array}$ & $\begin{array}{c}11 \\
61.1 \%\end{array}$ & $\begin{array}{c}6 \\
33.3 \%\end{array}$ & $\begin{array}{c}1 \\
5.6 \%\end{array}$ \\
\hline $\begin{array}{l}\text { 12. Lifelong Learning - } 27 \\
\text { indicators }\end{array}$ & $\begin{array}{c}2 \\
7.4 \% \\
\end{array}$ & $\begin{array}{c}23 \\
85.2 \%\end{array}$ & $\begin{array}{c}2 \\
7.4 \% \\
\end{array}$ \\
\hline
\end{tabular}

CEEA14; Paper 124

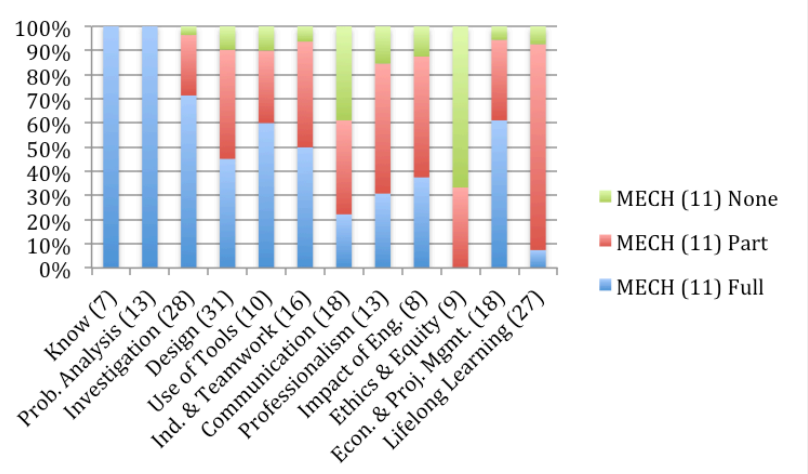

Fig. 4. Percentage of CEAB Graduate Attribute indicators assessed (Full), demonstrated (Part) and undemonstrated (None) in 11 courses in the Mechanical Engineering program, 2011-14.

\section{DISCUSSION}

It is important to realize that these data could be easily misinterpreted. Although on average 10 courses were assessed for each department, each instructor was expected to consider the manifestation and measurement of only 4 attributes per course. Therefore, the data really represent the treatment of the graduate attributes for an average of 3 courses per department. Considering the data in this light, the faculty can be pleased that on majority, only one attribute per department was not assessed in the courses that were considered for the study.

That being said, it is beneficial to ask what else these findings demonstrate. For the Biosystems engineering program, $50 \%$ or more of the indicators of three attributes were assessed in the selected courses: A Knowledge Base for Engineering, Problem Analysis, and Design. The indicators for the attribute, Impact of Engineering on Society and the Environment were not assessed in any of the Biosystems courses that were considered for the study, and a minimal number of indicators were assessed for Individual and Teamwork (below 10\%). More than $50 \%$ of the indicators for these attributes were not demonstrated: Individual and Teamwork, Impact of Engineering on Society and the Environment, and Ethics and Equity.

The findings for the Civil engineering program show that $50 \%$ or more of the indicators for the attributes, A Knowledge Base for Engineering, Problem Analysis, Investigation, Design, Use of Engineering Tools, Communication Skills, Impact of Engineering on Society and the Environment, and Economics and Project Management were assessed in the selection of courses. No indicators were assessed for the attribute, Lifelong Learning, and less than $10 \%$ of the indicators were assessed for the attribute, Ethics and Equity. More than $50 \%$ of the indicators for these attributes were not demonstrated: Professionalism, Ethics and Equity, and Lifelong Learning. 
Analysis of the findings for the Department of Electrical and Computer engineering confirmed that 50\% or more of the indicators for these attributes were assessed: A Knowledge Base for Engineering, Problem Analysis, Investigation, Use of Engineering Tools, and Economics and Project Management. For two attributes, Communication Skills, and Ethics and Equity, no indicators were assessed. Professionalism and Lifelong Learning indicators were minimally assessed (less than $10 \%$ ), and more than $50 \%$ of the indicators for both Communication Skills, and Ethics and Equity were not demonstrated.

The Mechanical engineering program courses that were scrutinized for the study exhibited six attributes whose indicators were assessed $50 \%$ or more: A Knowledge Base for Engineering; Problem Analysis; Investigation; Use of Engineering Tools; Individual and Teamwork; and Economics and Project Management. The indicators for the attribute Ethics and Equity were not assessed; and the indicators for Lifelong Learning were minimally assessed (less than 10\%). More than fifty percent of the indicators for Ethics and Equity were not demonstrated.

A holistic analysis of the data for all four departments reveals that overall, $50 \%$ or more of the indicators for the attributes, A Knowledge Base for Engineering and Problem Analysis were assessed. These attributes represent two of the five traditional skills of engineering [10][6]. Attributes whose indicators were not assessed at all included Communication Skills, Impact of Engineering on Society and the Environment, Ethics and Equity, and Lifelong Learning, all considered the professional skills of engineering [10][6]. Similarly, it was again the professional skills whose indicators were minimally assessed: Individual and Teamwork; Professionalism; Ethics and Equity; and Lifelong Learning. Finally, there was one attribute in all four departments where $50 \%$ or more of its indicators were not demonstrated, and that was the professional skill, Ethics and Equity. These findings reflect the engineering education research, which shows that not only is there less evidence of assessment of the professional skills, instructors find them more difficult to assess [8].

\section{NEXT STEPS}

This faculty attribute assessment study was a longitudinal study with the aim to explore how the 12 CEAB graduate attributes are manifest and measured in some of the engineering courses in the four engineering departments in the Faculty of Engineering at the University of Manitoba: Biosystems, Civil, Electrical and Computer, and Mechanical engineering. The findings from this study have given us some insight into where and how the graduate attributes are taught and assessed, and where they are less demonstrated in our engineering programs, and generally confirm that in these data, the traditional skills are more commonly assessed than the professional skills. This trend would be worth investigating further. As shown, it is heartening that in an average of three courses, the majority of the graduate attributes are assessed or demonstrated. However, increasing the demonstration and assessment of the professional skills in our programs would be an advisable endeavor, especially as embodying the professional skills are deemed critical to the success of today's engineer $[16][17][18][19]$.

At this point, we have a set of assessment data that departments can use for their individual assessment protocols. The data offer some valuable information, and it is suggested that departments triangulate them with other sources of available data. Alternate data include those from the student exit surveys [11][12][13], student forums [14], Industry Forums [15], and the course curriculum graduate attribute maps that were produced for the purposes of accreditation. For example, at the same time this study was being executed, an explanatory case study was being conducted to examine fourth year engineering student perceptions of the CEAB graduate attributes in the Mechanical Engineering program [13]. Findings from the study indicate that students would like the program to emphasize several professional attributes more, including Ethics and Equity and Professionalism. Students recommended holding mandatory seminars throughout the semester where Industry leaders would speak about authentic ethical and professional issues engineers face today. Additionally, it was recommended that students in the program be required to write a paragraph on environmental impact in every design report to address more comprehensively the attribute, Impact of Engineering on Society and the Environment. Thereby, once the data from this faculty attribute assessment study are triangulated with other assessment data, trends can be highlighted, and plans can be made within each department for next steps contingent on the results.

Concluding our previous paper for CEEA 2014 [8], which highlighted the findings in the data collected in Fall 2013, we wrote that the checklist in its present form would be retired due to the re-development of the indicators for each of the graduate attributes, all of which transpired with the creation of a set of graduate attribute rubrics [20]. However, it might be worthwhile to consider resurrecting Part A of the checklist and have instructors of the remaining courses in each department assess one set of four targeted graduate attributes. This would give an even more comprehensive picture of how the graduate attributes are manifest and measured in our engineering programs. Alternatively, every indicator does not necessarily need to be targeted for an attribute to be considered assessed. Further exploration of these data could determine the majority of indicators that academics are choosing, and an investigation could be conducted 
into how many indicators are considered 'enough' to assess students' graduate attribute outcomes.

In the end, we have enough data from this study, and from other sources of assessments, for individual departments to look at 'closing the loop.' In other words, it is time to consider using the results for the purposes of program improvement, a vital step in the CEAB accreditation mandate [5]. This is not easy. As stated by Kaupp and Frank:

Since 2012, the engineering education community has become comfortable with many aspects of the outcomes-based mandate, yet the continuous curriculum or program improvement side of the mandate is an area of much concern and question... Simply collecting the data of student performance and improving a single course on its own may be straightforward, but making meaning of the data and then effectively implementing a change across a program is far more complex. [5]

There is no singular way to go about closing the loop of program assessment [5]; ultimately, this will be a decision for faculty from each of our engineering departments to make. However, we are hopeful that these data, gathered with the cooperation of many of our faculty, will help point out the direction those decisions could take.

\section{CONCLUSION}

New work is continually being undertaken in the Faculty of Engineering at the University of Manitoba to explore outcomes-based assessment in our four engineering programs. Nevertheless, the findings from this longitudinal study have laid the groundwork for closing one loop of our program assessment efforts. Through three years of investigation, we have been provided with an overview of how the 12 graduate attributes are manifest and measured in the courses in our four engineering departments. There are clearly trends that support the research on the proclivity for assessing the traditional versus the professional skills in engineering education that should inform our program improvements.

Reflecting on this study has revealed its value, and the vision we have gained regarding our engineering programs and the $12 \mathrm{CEAB}$ graduate attributes. By closing this assessment loop, we will experience improvements in our engineering curriculum that will fully demonstrate our commitment to an outcomes-based engineering education assessment protocol.

\section{Acknowledgements}

The authors gratefully acknowledge all of our faculty instructors who participated in this study from 2011-2014, including the following instructors who worked with us during the Winter 2014 semester: Stefan Cenkowski, Nazim Cicek, Chuang Deng, Kathryn Atamanchuk, Ian Jeffrey, Behzad Kordi, Daniel Card, Tricia Stadnyk, Gregg Wyatt and David Levin. This study was made possible because of their valuable input. This research was supported by funding from Dr. Ingram's University of Manitoba Faculty of Engineering Start-up Grant, and Jillian Seniuk Cicek's University of Manitoba Graduate Fellowship (UMGF) and Manitoba Graduate Scholarship (MGS). The authors would like to thank the staff of the Dean's Office and the University of Manitoba NSERC Design Chair for their support.

\section{References}

[1] Linda Suskie, "Understanding the nature and purpose of assessment," in Designing Better Engineering Education Through Assessment. eds. J. E. Spurlin, S. A. Rajala, and J. P. Lavelle, 23-58. Sterling, VA: Stylus, 2008, 360 pp.

[2] Maddalena Taras, "Assessment - summative and formative some theoretical reflections," British Journal of Educational Studies, vol. 53, no. 4, pp. 466-478, 2005. Available as of October 3, 2014 from http://www.pgce.soton.ac.uk/IT/Teaching/Assessment/Tarras .pdf

[3] Marilee J. Bresciani, Outcomes-Based Academic and CoCurricular Program Review: A Compilation of Institutional Good Practices. Sterling, VA: Stylus, 2006, 214 pp.

[4] Amy Driscoll and Swarup Wood, Developing Outcomesbased Assessment for Learning-centered Education: A Faculty Introduction. Sterling, VA: Stylus, 2007, 275 pp. \{ISBN: 978 I 579221959$\}$

[5] Jake Kaupp and Brian Frank, "Approaching the loop: A brief review of effective practises in continuous program improvement, in Proc. CEEA Canadian Engineering Education Conf., CEEC14, Marjan Eggermont and Bill Rosehart (eds.) (Canmore, AB; 8-11 June 2014), 6 pp., 2011.

[6] Sandra Ingram, Jillian Seniuk Cicek and Nariman Sepehri, "The Attribute assessment process at the University of Manitoba," in Proc. CEAA Canadian Engineering Conf., CEEC12, (Winnipeg, MB; 17-21 June 2012), 7 pp., 2012.

[7] Jillian Seniuk Cicek, Sandra Ingram and Nariman Sepehri, "The attribute assessment process at the University of Manitoba: Year two," in Proc. CEEA Canadian Engineering Education Conf., CEEC13, Michel Perrier and Sylvie Dore (eds.) (Montreal, QC; 17-20 June 2013), 7 pp., 2013.

[8] Jillian Seniuk Cicek, Sandra Ingram and Nariman Sepehri, "The attribute assessment process at the University of Manitoba: Year three," in Proc. CEEA Canadian Engineering Education Conf., CEEC14, Marjan Eggermont and Bill Rosehart (eds.) (Canmore, AB 8-11 June 2013), 7 pp., 2014. 
[9] Jillian Seniuk Cicek, Sandra Ingram, and Nariman Sepehri, "Outcomes-based assessment in action: engineering faculty examine graduate attributes in their courses," International Journal of Engineering Education, vol. 30, no. 4, pp. 788805,2014

[10] Larry J. Shuman, Mary Besterfield-Sacre and Jack McGourty, "The ABET "professional skills" - Can they be taught? Can they be assessed?," Journal of Engineering Education, vol. 94, no. 1, pp. 41-55, 2005.

[11] Jillian Seniuk Cicek, Paul Labossiere, and Danny Mann, "Surveying fourth year engineering student perceptions of graduate attribute competencies," in Proc. CEEA Canadian Engineering Education Conf., CEEC13, Michel Perrier and Sylvie Dore (eds.) (Montreal, QC; 17-20 June 2013), 7 pp., 2013.

[12] Jillian Seniuk Cicek, Paul Labossiere and Sandra Ingram, "Examining fourth year engineering student perceptions of graduate attribute competencies: Year two," in Proc. CEEA Canadian Engineering Education Conf., CEEC14, Marjan Eggermont and Bill Rosehart (eds.) (Canmore, AB 8-11 June 2014), 9 pp., 2014.

[13] Jillian Seniuk Cicek, Paul Labossiere and Sandra Ingram, "Examining fourth year mechanical engineering student perceptions of graduate attribute competencies: Year three," (in press) in Proc. CEEA Canadian Engineering Education Conf., CEEC15, (eds.) (McMaster University 31 May - 3 June 2015), 8 pp., 2015.

[14] Dario Schor, Kathryn Marcynuk, Matthew Sebastian, Witold Kinsner, Ken Ferens, Cyrus Shafai and Nariman Sepehri, "Curriculum enhancement and evaluation of graduate attributes and outcomes through student-run forums," in Proc. CEEA Canadian Engineering Education Conf., CEEC11, Andy Fisher (ed.) (St. John's, NL; 6-8 June 2011), 6 pp., 2011.

[15] Ken Ferens, Jillian Seniuk Cicek, Nariman Sepehri, Witold Kinsner, J.P. Burak, Anne Parker, Dean McNeill, Douglas Ruth, Ian Jeffrey, Norma Godavari, and Sandra Ingram, "Industry Forum III: Towards a common language," in Proc. CEEA Canadian Engineering Education Conf., CEEC14, Marjan Eggermont and Bill Rosehart (eds.) (Canmore, AB; 8-11 June 2014), 7 pp., 2014.

[16] Honor Passow, "Which ABET competencies do engineering graduates find most important in their work?," Journal of Engineering Education, vol. 101, no. 1, pp. 95118, 2012.

[17] Thomas A. Litzinger, Lisa R. Lattuca, Roger G. Hadgraft and Wendy C. Newstetter, "Engineering education and the development of expertise," Journal of Engineering

Education, vol. 100, no. 1, pp. 123-150, 2011.

[18] Karl A. Smith, Sheri D. Sheppard, David W. Johnson and Roger T. Johnson, "Pedagogies of engagement: Classroombased practices," Journal of Engineering Education, vol. 94, no. 1, pp. 87-101, 2005.

CEEA14; Paper 124

-8 of $8-$
[19] Aman Yadav, Dipendra Subedi, Mary A. Lundeberg and Charles F. Bunting, "Problem-based learning: Influence on students' learning in an electrical engineering course,"

Journal of Engineering Education, vol. 100, no. 2, pp. 253280, 2011.

[20] Jillian Seniuk Cicek, Sandra Ingram, Nariman Sepehri, J.P Burak, Paul Labossiere, Danny Mann, Douglas Ruth, Anne Parker, Ken Ferens, Norma Godarvi, Jan Oleszkiewicz, and Aidan Topping, "Rubrics as a Vehicle to Define the Twelve CEAB Graduate Attributes, Determine Graduate Competencies, and Develop a Common Language for Engineering Stakeholders," in Proc. CEEA Canadian Engineering Education Conf., CEEC14, Marjan Eggermont and Bill Rosehart (eds.) (Canmore, AB; 8-11 June 2014), 6 pp., 2014. 